\title{
USOS DE LAS ENCUESTAS DE PRESUPUESTOS FAMILIARES
}

\author{
Vidal Díaz de Rada Igúzquiza
}

En este trabajo se va a realizar una contextualización del comportamiento de compra de las familias navarras utilizando el gasto en Bienes de Consumo y Servicios de las familias tal y como se desprende de la Encuesta de Presupuestos Familiares de 1991. La primera parte comienza con un análisis de los objetivos de este tipo de Encuestas y continúa con una exposición de cómo han evolucionado en la Comunidad Foral diversas magnitudes socio-económicas. Seguidamente se realiza una breve exposición del Análisis de Correspondencias como técnica que busca reducir una gran cantidad de datos a un conjunto de dimensiones latentes llamadas factores; para finalizar con la interpretación de los datos proporcionados por la Encuesta de Presupuestos Familiares de 1991(*).

\section{Introducción: definición y objetivos de las encuestas de presupuestos familiares}

Uno de los objetivos prioritarios de las Encuestas de Presupuestos Familiares es actualizar las ponderaciones de los distintos bienes y servicios que integran la «Cesta de la compra» para la elaboración del Indice de Precios al Consumo (IPC) (INE 1992: 7). Así, y aunque en su origen estas encuestas analizaban únicamente el gasto de las unidades familiares, la ampliación del objeto de estudio en las actuales encuestas obliga a recoger gran cantidad de información sobre las familias entrevistadas (número de miembros, origen de los ingresos, etc.), información que proporciona una visión detallada de los modos de vida al permitir

(*) El autor desea agradecer la gran ayuda recibida de la Delegación del Instituto Nacional de Estadística en Navarra. 
establecer conexiones entre los patrones de gastos, los ingresos de la unidad familiar, el equipamiento del hogar, las condiciones de las viviendas, etc. ${ }^{1}$.

En este sentido, y si consideramos que la estructura del gasto está condicionada por nuestra vida cotidiana, mediante el análisis de los patrones de gastos, podremos obtener una magnifica visión de los modos de vida de los individuos: analizando los gastos en ocio puede conocerse detalladamente el tipo de ocio, la frecuencia con la que se realizan ciertas actividades ociosas, etc. Este es el argumento esgrimido por numerosos científicos sociales cuando consideran que estas «elecciones (de compra) están limitadas únicamente por las necesidades objetivas de los individuos y sus recursos, la totalidad de la cultura material y las reglas de la economía política» (Sobel 1983: 521-523); de modo que cada individuo es el que configura y determina su propio estilo de vida, concepto que es definido como «conjunto de elecciones de comportamiento observables que los individuos hacen» (Sobel 1981, 1983). Este autor concede gran importancia a estas elecciones porque, junto a la ocupación, es uno de los elementos más importantes para determinar el prestigio social, además de ser más estable y observable que otros aspectos de la vida del individuo.

Pese al atractivo que ejerce realizar una investigación sobre los modos de vida a través de los patrones de gasto, este trabajo tiene un objetivo mucho más modesto como es contextualizar la cesta de la compra del consumidor navarro en la distribución de gastos de la sociedad española, a fin de conocer así las pautas diferenciadoras del consumidor navarro. No obstante, y antes de centrarnos en el objetivo propuesto, consideramos adecuado exponer las principales utilidades para las que se emplean las Encuestas de Presupuestos Familiares (Kantorowitz 1992: 113-114, Ballester Ríos 1984: 121-140):

1. Actualizar el IPC al ponderar la distribución del «cesto» de bienes y servicios, permitiendo conocer cómo cambian los hábitos de compra de las familias y así reducir la importancia ponderada de ciertos bienes que han visto reducido su consumo (por

1 Aunque el primer estudio científico sobre la distribución de los gastos de las familias fue realizado por William Petty en 1672 con objeto de establecer un nuevo sistema de impuestos (Petti 1899), no es hasta mediados del siglo XIX cuando se produce el gran desarrollo de las hoy llamadas «Encuestas de Presupuestos Familiares».

En España, los estudios sobre comportamientos de gasto comienzan en 1958 y desde entonces vienen realizándose cada diez años. Además de éstos, y con el objetivo de «conocer el origen y cuantía de la rentas familiares y su distribución entre los gastos de consumo», se viene realizando desde 1985 - y con una periodicidad trimestral - una Encuesta Continua de Presupuestos Familiares (ECPF). 
ejemplo, el gasto en correos), al tiempo que aumenta la ponderación de otros productos (gasto en teléfono, cintas de vídeo).

2. Proporcionar ponderaciones a fin de conocer el IPC de ciertos subgrupos específicos de población.

3. Conocer los lugares de realización de las compras al tiempo que se analizan los sectores comerciales en declive (pequeño comercio, por ejemplo) y otros en creciente desarrollo (hipermercados, etc.). Del mismo modo, esta información puede utilizarse para ajustar la muestra de establecimientos de IPC.

4. Examinar la relación existente entre los hábitos de consumo de las familias y sus características socio-económicas. Información que puede utilizarse para realizar análisis específicos de determinados colectivos de escasos recursos económicos, situaciones de pobreza, etc. (INE 1992: 7).

5. Conocer los niveles nutricionales de determinados subcolectivos sociales analizando el tipo de comida adquirida.

6. Examinar la elasticidad de la demanda de ciertos bienes.

7. Estudiar las condiciones de la vivienda y el equipamiento del hogar con relación al nivel de ingresos, el hábitat, etc.

8. Analizar detalladamente la distribución de los ingresos familares (rendimientos del trabajo, prestaciones sociales recibidas, etc.) y estudiar los hábitos de ahorro (tanto positivos como negativos) de ciertos subgrupos.

9. Averiguar las diferencias en el nivel de vida y en el coste de la vida entre determinadas partes del país, así como las diferencias entre el medio rural y urbano.

10. Examinar las variaciones en el nivel de vida en un determinado espacio temporal.

11. Con objeto de tomar decisiones comerciales y proveer las demandas de ciertos productos en determinados espacios geográficos.

12. Obtener información precisa para la elaboración de la Contabilidad Nacional (Azorín Poch-Sánchez Crespo 1986: 297-298).

13. Conocer la distribución de la renta (Rodríguez López 1977: 222-227).

\section{Análisis socio-económico de la sociedad navarra}

Una vez expuestas las principales utilidades de las Encuestas de Presupuestos Familiares procederemos a contextualizar el objeto de estudio mediante el análisis de la evolución socio-económica de la realidad na- 
varra: el desarrollo de Navarra hay que asociarlo - como el del resto del Estado- al Plan de Estabilización, aunque Navarra tardará 5 años en unirse a estas medidas económicas al aprobarse el 10 de abril de 1964 el Programa de Promoción Industrial de Navarra. En el año 1960 comienzan a producirse importantes cambios en la distribución de la población activa, aumentando poderosamente el volumen de empleo en la industria y los servicios y experimentando elevadas reducciones en el sector primario. J. Alcaide clasifica la evolución de la economía navarra en varios períodos (Alcaide y Cuadrado 1988: 19-26; Alcaide 1994: 2-24):

1. Período de Preestabilización (1955-1960): Caracterizado por un crecimiento económico inferior al de la economía española y un «peso» elevado del sector primario en la economía navarra.

2. El Boom económico (1960-1973): El sector agrario, pese a disminuir su ocupación, elevó considerablemente su productividad; la producción industrial creció a una tasa del 12,24\% como consecuencia de la implantación de industrias metálicas; el sector servicios aumentó poderosamente, aunque menos que en el resto de España.

3. De la crisis económica a la recuperación (1973-1985): En estos 12 años, caracterizados por fuertes desequilibrios a nivel internacional, la economía navarra creció a un ritmo inferior al $2 \%$ anual. Todos los sectores sufrieron fuertes reducciones de empleo, a excepción del sector servicios, que fue el dinamizador de la economía navarra al mostrar una evolución más favorable que el resto de los sectores (Rapún Gárate, 1990: 335-348).

4. En 1985 comienza un período de expansión económica que termina en 1990 y que coincide con un gran desarrollo económico en Europa y en el resto del Estado. Navarra, con un crecimiento del $7,1 \%$, fue la comunidad con una mayor expansión en este período (la media estatal fue de 5,6\%). Según Alcaide (1994: 5) la razón de este crecimiento se encuentra en el equilibrio de los sectores productivos.

5. El segundo año de la década de los 90 es el inicio de una etapa de recensión económica a nivel europeo que termina en 1993, recensión que afectó sobre todo a las Comunidades Autónomas que más crecieron en la etapa anterior (Alcaide 1994: 8). Aún así, el promedio del crecimiento navarro fue del $0,9 \%$, dos décimas por encima de la media estatal ${ }^{2}$.

2 Si se analiza la evolución de la economía desde 1986, Navarra es la comunidad de más crecimiento —-media 4,8\%—, con más de un punto por encima de la media estatal. 
6. A partir de $\mathbf{1 9 9 4}$ comienza a vislumbrarse una reactivación económica con una media de crecimiento del 3,35\% en Navarra y 2,26\% en el conjunto del Estado. El gran relanzamiento del sector industrial —con un crecimiento del 6,67\% — es el que más influencia ha tenido en la expansión de la economía navarra, seguido del sector servicios, que creció un 2,13\%. (Rapún y otros, 1995: 230.)

\section{A. Cambios en la distribución de la Población Ocupada y consecuencias derivadas}

Uno de los indicadores más directamente relacionados con el desarrollo de la economía es la evolución sectorial de la población ocupada: como puede observarse en el cuadro 1, desde 1967 hasta nuestros días ha cambiado tremendamente la composición de población ocupada en cada sector, consecuencia del proceso modernizador. En 1967 una de cada tres personas ocupadas trabajaba en el sector primario, mientras que catorce años más tarde (1981) este porcentaje se reduce a la mitad $(15,9 \%)$, y en 1991 tan sólo un 7,06\% de los empleos totales pertenecen al sector primario. Por otro lado, el sector servicios ha seguido una tendencia inversa puesto que a mediados de siglo ocupaba a un $28,9 \%$ de la población ocupada, y en 1991 da trabajo al 49,46\%. Mucho más irregular es la evolución del sector industrial, con una tendencia ascendente hasta la década de los 80 y una considerable estabilización de empleo a partir de esta fecha.

\section{Cuadro 1}

Distribución de los empleos 1967-1991

\begin{tabular}{lcccr}
\hline & $\begin{array}{c}1967 \\
\%\end{array}$ & $\begin{array}{c}1971 \\
\%\end{array}$ & \multicolumn{1}{c}{$\begin{array}{c}1981 \\
\%\end{array}$} & \multicolumn{1}{c}{$\begin{array}{c}1991 \\
\%\end{array}$} \\
\hline Agricultura & 31,71 & 28,04 & 15,90 & 7,06 \\
Industria & 39,31 & 40,40 & 43,50 & 43,47 \\
Servicios & 28,99 & 31,55 & 40,60 & 49,46 \\
\hline Pob. ocupada & $196.730,00$ & $198.658,00$ & $169.141,00$ & $195.113,00$ \\
\hline
\end{tabular}

Fuente: BBV, Renta Nacional de España y su Distribución Provincial, varios años.

Las alteraciones en la composición de la Población Activa no sólo tienen consecuencias económicas, sino que están íntimamente relacionados con los procesos de cambios residenciales, es decir, con los movimientos migratorios. El análisis de estos movimientos de población 
es muy importante en la medida que las necesidades de las personas van a estar condicionadas por el medio (rural/urbano) en el que viven, alterando así la demanda y la forma de adquirir bienes de consumo. Estos movimientos han incrementado el desequilibrio demográfico y económico de ciertas zonas, creando grandes problemas tanto en las zonas de emigración (con la pérdida de población joven) como en las de inmigración (al originar necesidades de equipamientos urbanos y sociales), modificando de este modo las demandas y necesidades sociales. En este sentido, hay que considerar dos fenómenos en el análisis de los procesos migratorios: la alteración de la población entre las regiones y el rápido proceso de urbanización de las ciudades.

\section{Distribución de la población entre regiones}

Los mayores movimientos de población se producen en España a partir de 1960 provocados por el gran desarrollo industrial de la década. Navarra, con un saldo migratorio negativo hasta el inicio de su industrialización, se encuentra con un triple proceso: por una parte un gran movimiento interno del campo a la ciudad, un proceso migratorio externo principalmente a Cataluña, Madrid y País Vasco, y una importante recepción de emigrantes de otras regiones a partir de 1960.

El inicio de la segunda ola de industrialización en 1960 cambia los procesos migratorios anteriores y Navarra pasa a ser receptora de emigrantes (Cuadro 2). Desde principios de siglo hasta 1960 Navarra había perdido 102.265 habitantes, y después de 1961 el proceso se invierte y pasa a ser receptora de emigrantes. No obstante, a partir de $1981-\mathrm{y}$ según datos del Anuario Estadístico de España- este proceso de recepción de inmigrantes se ralentiza considerablemente.

\section{Proceso de urbanización}

$\mathrm{Al}$ analizar el proceso de urbanización hay que considerar dos aspectos: la multiplicación de los puntos urbanos y el aumento de magnitud de cada uno de ellos. En los últimos 20 años el proceso de urbanización en España ha sido muy intenso, alterándose profundamente la distribución de la población en cuanto a la dimensión rural/urbana. En este sentido, Madrid y Cataluña se han caracterizado por un elevado grado de urbanización con una alta concentración de su población en grandes áreas metropolitanas, mientras que en Valencia y el País Vasco hay un predominio de ciudades medias, a pesar de tener grandes ciudades que superan los 10.000 habitantes. En comunidades como Aragón, Baleares, Cantabria, Navarra y La Rioja un gran centro urbano actúa como polo de atracción de la población (Del Campo y Navarro 1987: 115). 


\section{Cuadro 2}

Migraciones interprovinciales e intraprovinciales

\begin{tabular}{|c|c|c|c|c|c|c|}
\hline & \multicolumn{4}{|c|}{$\begin{array}{c}\text { Migraciones } \\
\text { interprovinciales }\end{array}$} & \multicolumn{2}{|c|}{$\begin{array}{c}\text { Migraciones } \\
\text { intraprovinciales }\end{array}$} \\
\hline & $1900-40$ & $1941-60$ & $1961-70$ & $1970-80$ & $1962-70$ & $1971-80$ \\
\hline Andalucía & -31.340 & -776.254 & -843.767 & -251.673 & 117.873 & 160.309 \\
\hline Aragón & -119.394 & -88.158 & $-34,352$ & -3.973 & 70.865 & 52.196 \\
\hline Baleares & +14.441 & +734 & +73.713 & +73.729 & 17.476 & 134.261 \\
\hline Canarias & +59.785 & -28.532 & +19.420 & +78.399 & 27.267 & 97.891 \\
\hline Cantabria & -39.003 & -50.389 & -14.485 & -117 & 19.609 & 23.921 \\
\hline Castilla-La Mancha & -172.983 & -412.646 & -458.532 & -203.974 & 53.622 & 61.138 \\
\hline Castilla-León & -536.048 & -485.190 & -466.403 & -186.199 & 143.422 & 130.823 \\
\hline Cataluña & +690.089 & +696.500 & +719.996 & +266.372 & 414.618 & 377.279 \\
\hline Com. Valenciana & +146.493 & -6.257 & +302.666 & +146.907 & 164.889 & 289.789 \\
\hline Extremadura & -68.312 & -201.608 & -378.165 & -161.122 & 36.646 & 41.935 \\
\hline Galicia & -240.131 & -352.702 & -229.167 & -3.626 & 40.665 & 66.700 \\
\hline Madrid & +611.392 & +637.220 & +686.554 & +342.059 & 60.327 & 366.930 \\
\hline Murcia & -93.175 & -125.646 & -101.651 & +9.891 & 10.708 & 19.641 \\
\hline Navarra & -61.933 & -40.332 & +18.510 & +2.064 & 38.370 & 35.545 \\
\hline País Vasco & +50.952 & +177.984 & +256.098 & +23.392 & 143.063 & 121.659 \\
\hline Pr. de Asturias & -22.740 & -80 & -31.345 & -9.978 & 17.826 & 39.873 \\
\hline La Rioja & -39.203 & -32.448 & -12.544 & +2.170 & 17.945 & 10.672 \\
\hline
\end{tabular}

Fuentes: Estudio sobre la Población Española, Tercer Plan de Desarrollo, Madrid, 1972. INE, Migración interior de España 1960-1970 y 1967-1975, Movimiento natural de la población 1976-1981, y Censo de población de 1981. Recogido de S. del Campo y M. Navarro, 1987: 86 y 91.

Cada uno de estos tres «tipos» de asentamientos ha condicionado poderosamente la estructura comercial de cada comunidad, al tiempo que ha introducido modificaciones en los hábitos de consumo de sus habitantes. En este sentido, mientras que en Valencia y el País Vasco - por sus peculiaridades residenciales - los comercios están relativamente diseminados por una gran cantidad de ciudades medias, comunidades como Navarra se caracterizan por un centro urbano con una concentración de establecimientos especializados en Textil, Calzado y Farmacia-Droguería, y una amplia presencia de otro tipo de establecimientos comerciales repartidos por toda la geografía navarra. Qué duda cabe de que la existencia de zonas comerciales altamente equipadas (Logroño, Calahorra, Zaragoza, Vitoria, San Sebastián, Bilbao) muy próximas a un gran número de núcleos rurales están reduciendo el poder de atracción comercial de la capital. 
Cuadro 3

Relación entre población y establecimientos minoristas

\begin{tabular}{lcrrrrrrr}
\hline & Total & Zona I & Z. II & Z. III & Z. IV & Z. V & Z. VI & Z. VII \\
\hline \% Población & 100 & 10,63 & 2,94 & 49,21 & 7,08 & 5,93 & 10,34 & 13,87 \\
\% Comercios & 100 & 8,98 & 2,17 & 49,91 & 6,06 & 6,92 & 9,93 & 16,03 \\
Alimentación & 100 & 7,80 & 1,53 & 49,84 & 5,37 & 7,69 & 11,65 & 16,12 \\
Textil & 100 & 6,32 & 0,75 & $\mathbf{5 5 , 9 4}$ & 5,24 & 5,66 & 8,82 & 17,72 \\
Calzado & 100 & 6,21 & - & $\mathbf{5 7 , 9 9}$ & 5,92 & 4,44 & 8,28 & 17,16 \\
Farmacia-Droguería & 100 & 7,72 & 2,12 & $\mathbf{5 8 , 3 0}$ & 6,56 & 5,79 & 7,72 & 11,79 \\
Equip. hogar & 100 & 8,74 & 0,90 & 47,79 & 5,62 & 7,53 & 10,24 & 19,18 \\
Vehículos & 100 & 12,37 & 2,89 & 36,32 & 8,95 & 7,10 & 10,79 & 21,58 \\
Mixto & 100 & 21,94 & 9,47 & 26,02 & 10,07 & 8,88 & 12,71 & 10,91 \\
Otros & 100 & 5,63 & 1,33 & 61,18 & 4,80 & 5,79 & 6,37 & 14,90 \\
\hline
\end{tabular}

Z.I: Noroeste, Z.II: Pirineos, Z.III: Pamplona, Z.IV: Tierra Estella, Z.V: Media Oriental, Z.VI: Ribera Alta, Z.VII: Tudela.

Fuente: Gobierno de Navarra, Panorama estadístico de Navarra, 1990, p. 134.

El cuadro anterior, que compara las personas residentes en cada zona y los establecimientos minoristas de éstas, apenas informa sobre la distribución residencial de la población navarra con relación al tamaño del municipio de residencia. Con este fin se ha elaborado el gráfico 1 , en el cual puede apreciarse el gran aumento de población experimentado en los municipios mayores de 5.000 habitantes, provocando la pérdida de población en los núcleos más pequeños. Mientras que en 1900 únicamente uno de cada cinco navarros vivía en poblaciones mayores de 5.000 habitantes, en 1970 la mitad de la población reside en estos municipios y en 1994 el 61,1\%.

Por otro lado, y según estudios recientes sobre Diferenciación Residencial (Leonardo Aurtenetxe 1989, Castells 1986, Del Campo 1983: 137-153), dentro de las ciudades hay fuertes movimientos residenciales que producen, entre otras consecuencias, una especialización del centro de la ciudad en área funcional y administrativa mientras que la periferia adopta una función residencial ${ }^{3}$. Consecuentemente, la escasa población que reside en el centro tiene una edad elevada, mientras que en la periferia está compuesta principalmente por personas jóvenes ${ }^{4}$.

3 Es el caso del importante aumento de población en los últimos años de determinados municipios de la Comarca de Pamplona como Zizur, Egües, Mendillorri, etc.

4 De este modo, los hipermercados situados en la periferia de Pamplona y muy cercanos a la red viaria, atraen fundamentalmente a las personas que viven en estas áreas resi- 


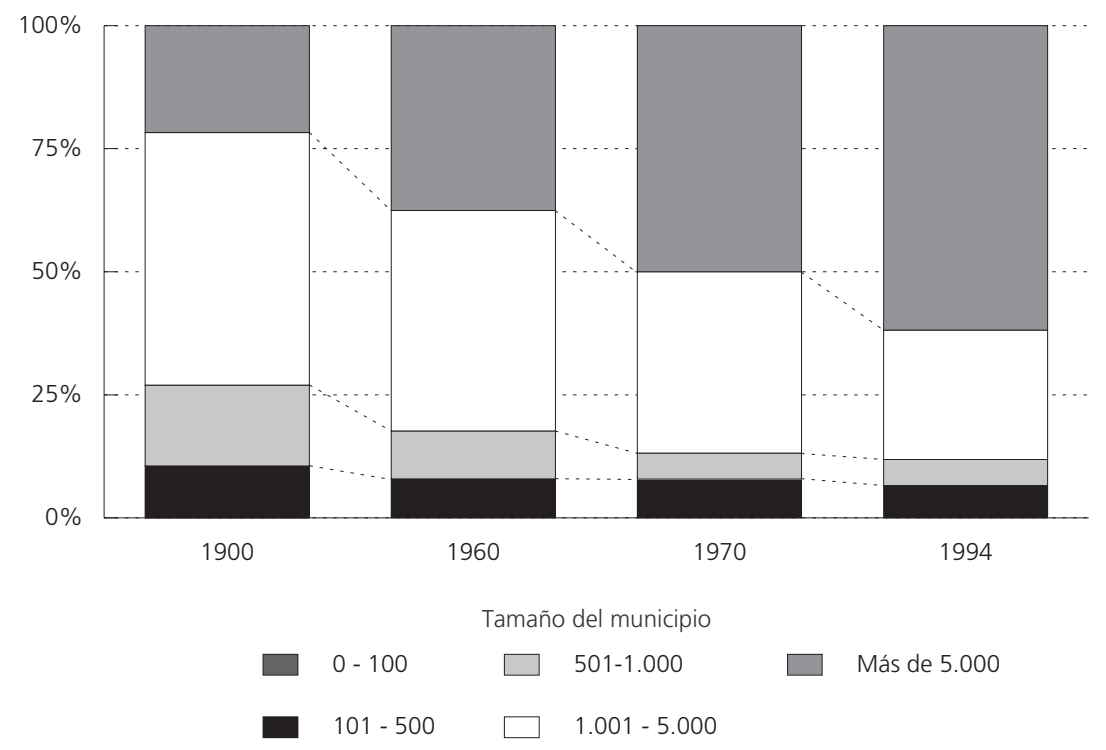

Fuente: Gobierno de Navarra, Población de los Ayuntamientos y Concejos de Navarra de 1900 a 1986. En 1994: Navarra en Cifras 1994, p. 9.

\section{Gráfico 1}

Distribución de la población navarra según el tamaño de los municipios

\section{B. Evolución de la Renta Familiar Disponible en Navarra}

Otra de las variables importantes en la definición del consumidor es la Renta Familiar Disponible: como puede observarse, el análisis del cuadro 4 permite comparar la evolución de la Renta Familiar Disponible en Navarra y en el resto del Estado durante el período 1967-1991. En 1967 la Renta por individuo era notablemente superior a la media nacional, aunque al comparar la evolución en los dos espacios geográficos

denciales debido a los escasos costes de desplazamiento y la rapidez en la accesibilidad; descongestionando así el área comercial del centro de la ciudad y el comercio existente en estas áreas residenciales (Mújica, 1995: 83-85). En coherencia con este planteamiento, el cliente predominante del Hipermercado tiene una edad intermedia (25-40 años), casado, trabaja, con estatus medio-alto y vive en hogares de entre 4 y 6 personas. (Perfil perteneciente al cliente del Hipermercado en la CAPV, según se desprende de Gobierno Vasco, 1994: 90). 
puede apreciarse cómo hasta finales de la década de los setenta esta magnitud aumenta menos en Navarra que en el resto del Estado. No obstante, en los ochenta el incremento porcentual en Navarra vuelve a ser ligeramente superior a la media nacional, de modo que en el año 1991 la RFD per cápita en Navarra supera a la media nacional en $13.483,5$ pesetas (de 1967).

\section{Cuadro 4}

Evolución de la renta familiar disponible entre 1967 y 1991

\begin{tabular}{|c|c|c|c|c|c|}
\hline & \multicolumn{2}{|c|}{ Navarra } & \multicolumn{2}{|c|}{$\begin{array}{l}\text { Conjunto } \\
\text { Nacional }\end{array}$} & \multirow{2}{*}{$\begin{array}{c}\text { Indice } \\
\text { Comparado } \\
\begin{array}{c}\text { Navarra/Resto } \\
(* *)\end{array}\end{array}$} \\
\hline & $\begin{array}{l}\text { En ptas. } \\
\text { de } 1967\end{array}$ & $\begin{array}{c}\text { Incremento } \\
\text { porcentual }(*)\end{array}$ & $\begin{array}{l}\text { En ptas. } \\
\text { de } 1967\end{array}$ & $\begin{array}{c}\text { Incremento } \\
\text { porcentual }(*)\end{array}$ & \\
\hline 1967 & $49.424,0$ & & $32.455,0$ & & 118,0 \\
\hline 1971 & $61.787,6$ & $25,0 \%$ & $53.448,5$ & $64,7 \%$ & 117,0 \\
\hline 1975 & $69.889,9$ & $13,1 \%$ & $64.044,6$ & $19,8 \%$ & 109,0 \\
\hline 1977 & $69.126,8$ & $0,9 \%$ & $64.574,8$ & $0,8 \%$ & 109,0 \\
\hline 1979 & $69.059,6$ & $1,1 \%$ & $67.005,5$ & $3,8 \%$ & 103,0 \\
\hline 1981 & $69.837,8$ & $1,1 \%$ & $68.069,9$ & $1,6 \%$ & 102,0 \\
\hline 1983 & $73.584,0$ & $5,4 \%$ & $68.231,8$ & $0,2 \%$ & 107,0 \\
\hline 1985 & $70.573,2$ & $0,9 \%$ & $67.904,1$ & $0,9 \%$ & 103,0 \\
\hline 1987 & $78.980,5$ & $19,1 \%$ & $76.161,4$ & $13,0 \%$ & 104,0 \\
\hline 1989 & $87.877,6$ & $11,3 \%$ & $83.198,7$ & $8,4 \%$ & 106,0 \\
\hline 1991 & $101.146,7$ & $15,1 \%$ & $87.663,2$ & $5,3 \%$ & 115,4 \\
\hline
\end{tabular}

(*) $\frac{\text { Año } X}{\text { Año } X-1}=100$

(**) Base media nacional $=100$

Fuente: Banco de Bilbao, Renta Nacional de España y su distribución provincial.

No nos detendremos aquí en analizar las razones que han provocado que la renta sea superior en Navarra, aunque sí queremos hacer notar que esta mayor disposición de ingresos se verá reflejada en un incremento del gasto de sus habitantes. Por otro lado, estos elevados niveles de renta han provocado un aumento del nivel educativo en la Comunidad Foral, tal y como se desprende del examen de los conceptos del cuadro 5: en 17 años (1974-93) se ha triplicado el número de personas que cursan estudios universitarios, de alumnos matriculados en Formación Profesional, y de personas con título universitario. 


\section{Cuadro 5}

Nivel educativo en Navarra

(Por 1.000 habitantes)

\begin{tabular}{lrrr}
\hline & 1974 & 1984 & 1991 \\
\hline Personas que cursan estudios & 227,2 & 244,5 & 259,45 \\
Alumnos matriculados en BUP/COU & 29,2 & 30,2 & 43,26 \\
Alumnos matriculados en FP & 8,1 & 22,8 & 22,73 \\
Personas que cursan estudios univers. & 7,8 & 13,2 & $24.18^{*}$ \\
Personas con título univer./ & & & \\
Téc.Sup. que no están cursando estudios & 12,3 & 22,6 & 33,61 \\
\hline
\end{tabular}

(*) Recoge los conceptos «Arquitectura/Ingeniería Técnica», «Facultades o Colegios Superiores» $\mathrm{y}$ «Escuelas Técnicas Superiores».

Fuente: En 1974 y 1984: A. Sanz y M. de Teran, «Navarra, a través de los indicadores sociales», 1988. En 1991: Censo de Población y Vivendas.

El análisis de otros indicadores puede aportar una imagen más exacta de la realidad cultural navarra. El índice de difusión de la prensa es el segundo más alto de España, con 158,2 periódicos por 1.000 habitantes, y la media de revistas de información generales ${ }^{5}$ es de ocho revistas por habitante.

\section{C. Evolución de los gastos de las familias navarras: las encuestas de presupuestos familiares}

Si bien los indicadores utilizados con anterioridad pueden proporcionarnos una panorámica de la sociedad navarra, serán los resultados de las Encuestas de Presupuestos Familiares los que nos proporcionarán una visión más exacta para nuestros objetivos. Tal y como se desprende del cuadro 6, en el año 1964-65 el 42,7\% de los gastos de las familias se dedicaban a productos de alimentación, y un $11 \%$ en «Gastos diversos», en los que se incluyen conceptos como el aseo personal, sanidad, transportes y comunicaciones, gastos en cultura y en tabaco. Diez años más tarde el porcentaje de gastos en alimentación continúa siendo el mismo, y los gastos en vivienda, calefacción y alumbrado llegan al $16 \%$.

5 En este apartado se consideran todo tipo de revistas, desde las llamadas revistas del corazón (Hola, Semana, etc.), y revistas especializadas (El mueble, Mundo y Hogar, Moto Verde, etc.) hasta revistas científicas (Muy interesante, etc.). 
Los servicios médicos reducen ostensiblemente su participación en la distribución de gastos familiares como consecuencia de la redacción, el 28 de diciembre de 1963, de un Proyecto de Ley en el que se formula un «Plan Nacional de Seguridad Social» sustituyendo la Previsión Social, con seguros sociales aislados y heterogéneos, por un sistema moderno de Seguridad Social (Del Val y de la Fuente, 1986: tomo I, p. 9.2).

\section{Cuadro 6}

Evolución de los gastos en los hogares navarros

(Gasto anual por persona)

\begin{tabular}{|c|c|c|c|c|}
\hline & $1964-1965(* *)$ & 1975 & $1980-81$ & $1990-91$ \\
\hline 1. Alimentos, bebidas y tabaco & 42,7 & 42,2 & 29,2 & 24,0 \\
\hline 2. Vestido y calzado & 15,4 & 10,0 & 9,0 & 12,1 \\
\hline 3. Vivienda, calefacción & 71 & 162 & 169 & 94 \\
\hline 4. Muebles, enseres y & & & & \\
\hline servicios del hogar & 11,1 & 8,6 & 8,1 & 8,2 \\
\hline $\begin{array}{l}\text { 5. Serviciós médicos y } \\
\text { gastos sanitarios }\end{array}$ & 23,2 & 2,7 & 2,5 & 3,0 \\
\hline 6. Transportes y comunicaciones & 0,5 & 7,8 & 15,5 & 15,2 \\
\hline $\begin{array}{l}\text { 7. Esparcimiento, enseñanza } \\
\text { y cultura }\end{array}$ & & 4,4 & 7,0 & 6,9 \\
\hline 8. Otros bienes y servicios & & 2,3 & 8,5 & 15,3 \\
\hline $\begin{array}{l}\text { 9. Otros gastos no mencionados } \\
\text { anteriormente }\end{array}$ & & 5,7 & 3,2 & 5,8 \\
\hline Total & $24.804,0$ & $78.206,0$ & $286.208,0$ & $764.335,0(*)$ \\
\hline
\end{tabular}

(*) Gasto total en pesetas de cada año.

(**) En el año 1964-65 el grupo 3 únicamente comprende «Gastos de alquiler y reparación de la vivienda», y en el grupo 4 están incluidos «Gastos de la casa» como calefacción, alumbrado, muebles, serv. doméstico, etc. En el grupo 5 están clasificados los «Gastos diversos» y en el 6 las «Vacaciones».

Fuente: INE, Encuestas de Presupuestos Familiares, varios años.

Sin embargo, no es hasta el año 1980-81 cuando se produce un enorme cambio en los hábitos de compra de las familias navarras, comenzando a surgir un consumo característico de países desarrollados. Únicamente el 29,2\% de la renta se dedica a la alimentación, aumentando otras partidas de gasto como los transportes y comunicaciones, «Esparcimiento, enseñanza y cultura», y «otros bienes y servicios» (Martín Guzmán 1990: 304-309). Alfonso Rebollo, en un análisis de la estructura del consumo de las economías domésticas en el conjunto nacional, señala que la estructura del gasto en consumo de los españoles 
en 1980 es la propia de un país industrializado caracterizado por un descenso del gasto en alimentación (alrededor de 30\%), estabilización de los gastos en vestido y calzado (sobre el 10\%) y una mayor importancia relativa concedida a los transportes y comunicaciones, cultura y otros bienes y servicios (Rebollo Arévalo, 1983: 44-45).

En el estudio realizado en 1990-91 se aprecia una disminución del

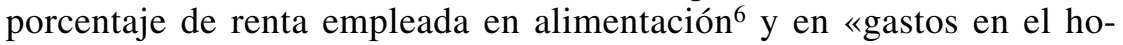
gar», aumentando los gastos en vestido, calzado y en «otros bienes y servicios». Conviene tener en cuenta la distribución de los gastos familiares del año 1991, ya que éste va a ser el punto de partida de la presente investigación.

Por otro lado, las encuestas de Presupuestos Familiares también aportan información sobre el nivel de equipamiento y el estado de los hogares. Según se desprende de la encuesta realizada en el año 1991, Navarra se encontraba entre las 5 regiones más prósperas contabilizando el número de automóviles, lavadoras automáticas, lavavajillas, teléfonos y ordenadores personales (Cuadro 7).

\section{Cuadro 7}

Niveles de equipamiento y condiciones de las viviendas (año 1991)

\begin{tabular}{lccrrrrr}
\hline & $\begin{array}{c}\text { Auto- } \\
\text { móviles }\end{array}$ & $\begin{array}{c}\text { TV en } \\
\text { color }\end{array}$ & $\begin{array}{c}\text { Lavad. } \\
\text { Automát. }\end{array}$ & $\begin{array}{c}\text { Lava- } \\
\text { vajillas }\end{array}$ & $\begin{array}{c}\text { Conge- } \\
\text { lador }\end{array}$ & $\begin{array}{c}\text { Ordenador } \\
\text { personal }\end{array}$ & Teléfono \\
\hline Prósperas: & & & & & & & \\
$\quad$ Navarra & 73,5 & 96,7 & 96,5 & 16,4 & 11,3 & 9,3 & 87 \\
$\quad$ Cataluña & 67,1 & 96,2 & 95,0 & 12,0 & 7,1 & 16,6 & 89 \\
Baleares & 73,4 & 96,5 & 86,5 & 6,1 & 24,0 & 8,9 & 84 \\
$\quad$ Valencia & 70,3 & 96,6 & 90,2 & 6,5 & 2,2 & 9,8 & 76 \\
$\quad$ Madrid & 67,4 & 97,4 & 95,4 & 17,0 & 1,0 & 16,2 & 94 \\
País Vasco & 63,0 & 94,5 & 96,4 & 13,8 & 4,8 & 11,8 & 91 \\
Menos prósperas: & & & & & & & \\
$\quad$ Extremadura & 51,6 & 82,4 & 79,2 & 4,4 & 2,7 & 4,0 & 57 \\
Castilla-Man. & 57,1 & 90,4 & 86,4 & 5,0 & 0,9 & 6,2 & 69 \\
$\quad$ Andalucía & 55,0 & 92,1 & 88,0 & 5,4 & 3,3 & 9,4 & 61 \\
Castilla-León & 59,1 & 86,5 & 88,1 & 9,3 & 8,2 & 8,6 & 74 \\
$\quad$ Galicia & 61,5 & 75,2 & 81,6 & 6,4 & 29,0 & 5,8 & 64 \\
\hline
\end{tabular}

En porcentajes.

Fuente: INE, Encuesta de Presupuestos Familiares, año 1991.

${ }^{6}$ Después de Madrid, Navarra es la segunda comunidad que menos gasta en el concepto «Alimentación, Bebidas y Tabaco». 
Si se adopta una perspectiva más sintética y general que deja de lado la referencia a las Encuestas de Presupuestos Familiares, es preciso indicar que en 1988 se realizó un análisis a fin de medir el desarrollo autonómico a través de treinta indicadores clasificados en cinco grupos: Indicadores de Población, Sanitarios, Educación-Cultura, Vivienda y Equipamiento del Hogar. Las cuatro regiones mejor situadas fueron, por orden de importancia, Madrid, País Vasco, Navarra y Cataluña (Sanz y Teran 1988: 82-112).

A modo de conclusión, podría caracterizarse el entorno socio-geográfico objeto de estudio (Navarra) como una de las Comunidades más desarrolladas y prósperas del Estado; con un predominio de población ocupada en los servicios, una Renta Familiar superior al resto de España y una estructura de consumo caracterizada por emplear únicamente el $25,4 \%$ del gasto familiar en la compra de alimentos, bebidas y tabaco.

\section{La cesta de la compra del consumidor navarro en relación con la distribución de gastos en la sociedad española}

\section{A. Planteamiento metodológico}

Conocidos los principales usos de las las Encuestas de Presupuestos Familiares y una vez expuesta la evolución de la sociedad navarra en los últimos 30 años, procederemos a contextualizar la «cesta de la compra» del consumidor navarro según sus patrones de gasto en relación con el resto de las Comunidades Autónomas, seleccionando - de todos los datos aportados por la Encuesta de Presupuestos Familiaresaquellos que muestran el gasto anual medio por hogar según los grandes grupos de gasto en cada una de las Comunidades Autónomas durante los doce meses comprendidos entre abril de 1990 y marzo de 1991; que responde al período de tiempo analizado por la EPF de 1990-91. Realizada esta aclaración, se ha utilizado el Análisis Factorial de Correspondencias como método de selección, que «responde a la necesidad de profundizar en el conocimiento de las relaciones que se establecen entre dos variables cualitativas observadas en una misma población», insistiendo más concretamente en la explicación de cómo los distintos valores o categorías de ambas variables se relacionan unos con otros (García Santesmases 1984: 75). Dicho de otro modo, se trata de «recolocar» las filas y las columnas en un espacio de F dimensiones a fin de conocer si existen relaciones entre ellas; relaciones que serán utilizadas para definir los factores, analizar las filas y 
las columnas «raras», conocer la diferencia entre unas y otras, comprobar el «peso» que ciertas variables tienen en la definición de cada eje, etc. (Calvo Gómez 1993: 304).

Varios han sido los criterios que han incidido en la elección de esta técnica estadística, teniendo presente siempre el objetivo perseguido por este trabajo. Así, una vez consideradas las características de las distintas técnicas estadísticas que tienen como objeto de estudio realizar clasificaciones de la realidad observada (Análisis de Cluster, Escalas Multidimensionales, Componentes Principales, etc.) (Andrews, 1981), ha sido elegido el Análisis de Correspondencias atendiendo - fundamentalmente- a las limitaciones de los datos aportados, que no siguen una distribución normal, y que más bien hacen referencia a lo que es una tabla de contingencia entre provincias y grupos de gasto; siguiendo una afirmación expresada por F. Alcantud cuando señala que «la experiencia nos enseña que al analizar este tipo de datos utilizando el Análisis Factorial de Correspondencias en lugar de otros métodos como el Componentes principales se produce una mejora notable en los resultados, sobre todo cuando la variable, si no tiene una distribución normal, al menos es continua» (Alcantud 1992: 12, 43). Antes de finalizar, señalemos que consideramos que una exposición detallada de esta técnica no es posible ni adecuada en el marco del presente trabajo; por ello nos hemos limitado a exponer una idea sintética de las características de ésta, remitiendo al lector interesado a la bibliografía expuesta al final del trabajo.

\section{B. Interpretación de resultados}

Aplicando el Análisis de Correspondencias obtenemos dos factores que explican el $76 \%$ de la inercia total. Los tipos de gastos definitorios del primer factor, junto con las Comunidades Autónomas en los que predominan, aparecen expuestos en dirección horizontal en la figura I. Este primer factor, que explica el $56,7 \%$ de la inercia total, está señalando una oposición entre los gastos en SERVICIOS MEDICO-SANITARIOS Y TRANSPORTES-COMUNICACIONES, frente al gasto en ALIMENTOS, BEBIDAS Y TABACO.

En relación con el resto del panorama nacional el gasto en Alimentos, Bebidas y Tabaco es superior en Ceuta, Andalucía, Castilla-La Mancha, Extremadura, Galicia y Murcia. En el extremo izquierdo del factor se sitúan Comunidades Autónomas con un elevado gasto en servicios Médicos-Sanitarios y Transportes-Comunicaciones (Baleares, Navarra, País Vasco, Comunidad Valenciana y Cataluña), que emplean 


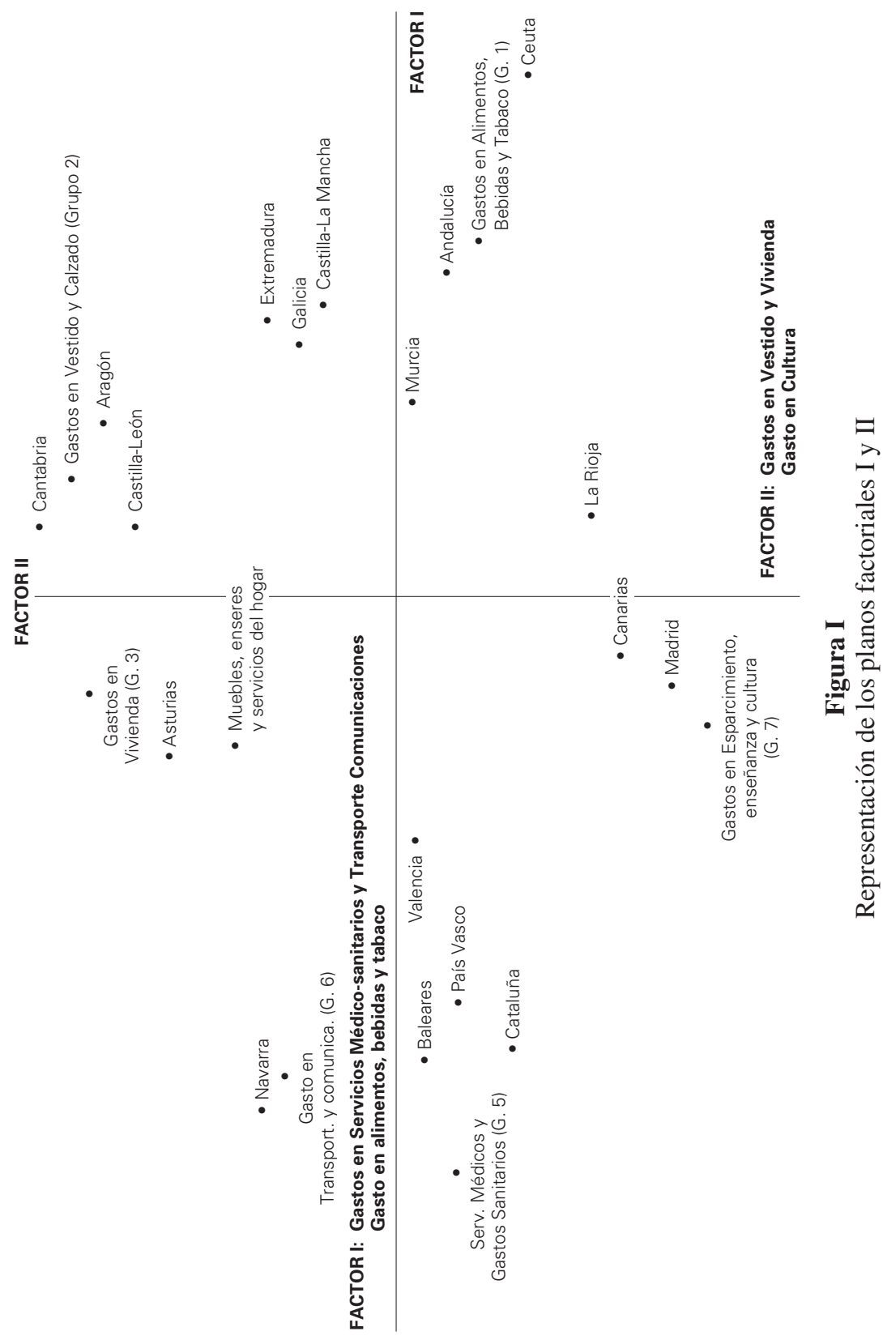


respectivamente en estos conceptos el $20 \%, 23 \%, 18 \%, 17 \%, 17,8 \%$ y $17 \%$ de todos los ingresos no ahorrados. La interpretación de estos resultados debe hacerse recordando la Ley de consumo de Ernest Engel: «la proporción del presupuesto que un consumidor emplea en bienes y servicios está afectada por las variaciones de su renta» (Houthakker, 1974: 251-252). Con objeto de comprobar la vigencia de esta proposición analizaremos si existe relación entre la agrupación realizada en este trabajo y la Renta Familiar Disponible de cada una de las Comunidades Autónomas. En el año 1991, y según datos procedentes del BBV y recogidos por Julio Alcaide (1993: 2-17), las comunidades con más Renta Familiar Disponible, por orden de importancia, son: Cataluña, Baleares, La Rioja, Madrid, Comunidad Valenciana y Navarra. De éstas, todas a excepción de La Rioja y Madrid (que serán analizadas seguidamente) forman parte de la clasificación realizada por este primer factor. Un comentario especial merece el País Vasco, caracterizado por emplear un alto porcentaje de sus gastos familiares en servicios médico-sanitarios y en transporte-comunicaciones, y con una Renta Familiar Disponible que ocupa, en 1991 , el $11^{\circ}$ puesto en la clasificación nacional. El gran nivel de desarrollo que tradicionalmente ha caracterizado a esta comunidad ha generado una «inercia» en los hábitos de gasto que se ha mantenido pese al gran descenso de la Renta percápita experimentado en los últimos años.

Frente a este consumo de servicios y bienes «discreccionales», en el otro extremo del factor se encuentran Comunidades Autónomas con baja Renta Familiar Disponible y que emplean una gran cantidad de sus ingresos en Alimentación, Bebidas y Tabaco. Utilizando la fuente citada anteriormente, las Comunidades Autónomas con menor renta son, por orden de importancia: Ceuta, Extremadura, Andalucía, Castilla La Mancha, Canarias y Galicia.

El análisis de las dimensiones verticales de la figura I nos muestra cómo el eje o Factor II completa y matiza lo ofrecido por el primero, ya que añade otra caracterización de gastos a la explicación anterior. Si el Factor I definía dos comportamientos atendiendo fundamentalmente al gasto en alimentación y en servicios médicos, en éste son los gastos en VESTIDO Y VIVIENDA, frente al gasto en CULTURA los que establecen unas diferencias más precisas. En la parte superior del factor aparecen Cantabria, Aragón, Asturias y Castilla-León caracterizadas por un elevado gasto, comparativamente hablando, en Vestidos, Vivienda y Mobiliario del Hogar. Así, la ley de Engel vuelve a ser útil para interpretar estos resultados, ya que las Comunidades Autónomas con más renta (Madrid y La Rioja) gastan una mayor cantidad de su presupuesto familiar en cultura. En el otro extremo, y a excepción de Ara- 
gón, con una RFD algo superior a la media española, el resto de las comunidades tienen una renta Percápita inferior a la media española, y han experimentado un considerable descenso en el período 1985-1991 (Alcaide Inchausti, 1992: 2-33).

Por otro lado, la «localización» de las Comunidades Autónomas en las diferentes cuadrículas - generadas por la intersección de los factores- está indicando la importancia relativa de otros gastos: así, comunidades como Cataluña y País Vasco, situadas en la parte izquierda inferior del factor I, caracterizadas por un consumo en Servicios Médicos y Transportes, destacan también por un elevado gasto en esparcimiento, enseñanza y cultura; mientras que otras comunidades como Navarra - situada en la cuadrícula superior izquierda del Factor I- emplean una parte destacada de su renta en vivienda y mobiliario. No obstante, debe tenerse en cuenta que el Gasto en Servicios Médicos y Transportes-Comunicaciones es el elemento diferenciador que caracteriza a estas comunidades, y el análisis de las cuadrículas únicamente proporciona una información complementaria.

\section{Reflexión final}

El objetivo de este trabajo ha sido realizar una descripción de las pautas diferenciadoras del consumidor navarro, tal y como se desprende de la Encuesta de Presupuestos Familiares de 1991. Antes de darlo por concluido, consideramos que puede ser de gran ayuda resumir en unas breves notas los hallazgos más interesantes:

-Pese a que el objetivo principal de las Encuestas de Presupuestos Familiares es actualizar las ponderaciones de los bienes y servicios para la elaboración del Indice de Precios al Consumo (IPC), en la actualidad recogen gran cantidad de información sobre las condiciones de vida de las familias entrevistadas: ingresos de la unidad familiar, origen de los ingresos, equipamiento del hogar, condiciones de las viviendas, etc.

-El aspecto más reseñable, tras aplicar el Análisis Factorial de Correspondencias a los datos suministrados por la Encuesta de Presupuestos Familiares, es la gran relación existente entre los elevados niveles de Renta Familiar Disponible y el descenso de los gastos en alimentación. Derivado de este planteamiento, llama la atención la aplicabilidad de la ley de Engelen en la realidad española, ya que las provincias con mayor Renta Familiar Disponible dedican una mayor proporción de sus gastos a Trans- 
portes y Comunicaciones, Servicios Médicos, y a Esparcimiento y Cultura.

- Navarra, pese a ser una comunidad que gasta algo más que la media nacional (cuadro 8) en Transportes y Comunicaciones, no aparece cercana a ninguna otra en la representación gráfica de los ejes del Análisis de Correspondencias, tal y como queda reflejado en la figura I. La explicación a este fenómeno puede encontrarse al analizar su localización en talos ejes: por un lado se encuentra en la parte izquierda del factor I, pero su presencia en la cuadrícula superior indica una originalidad no compartida por ninguna otra comunidad, ya que las familias navarras emplean un $20 \%$ de sus recursos en gastos de Vestido-Calzado $(12,1 \%)$ y en Muebles-enseres-servicios del hogar $(8,4 \%)$; alejándose así de los gastos en Esparcimiento-enseñanza y cultura, ámbito que le correspondería por tener una renta similar a comunidades como Madrid, Cataluña, Baleares, La Rioja y Comunidad Valenciana.

\section{Cuadro 8}

Distribución de gastos familiares comparados entre Navarra y el Conjunto Nacional (año 1990-91)

(Por persona y año)

\begin{tabular}{lrr}
\hline & Navarra & España \\
\hline 1. Alimentos, bebidas y tabaco & 24,0 & 28,3 \\
2. Vestido y calzado & 12,1 & 11,2 \\
3. Vivienda, calefacción y alumbrado & 9,4 & 10,1 \\
4. Muebles, enseres y servicios del hogar & 8,4 & 6,3 \\
5. Servicios médicos y gastos sanitarios & 3,0 & 3,1 \\
6. Transportes y comunicaciones & 15,2 & 14,2 \\
7. Esparcimiento, enseñanza y cultura & 6,9 & 7,1 \\
8. Otros bienes y servicios & 15,3 & 14,8 \\
9. Otros gastos no mencionados anteriormente & 5,7 & 4,9 \\
\hline
\end{tabular}

Fuente: INE, Encuesta de Presupuestos Familiares 1990-91, Madrid 1992.

- Por lo expuesto hasta el momento, sigue vigente la afirmación propuesta por A. Rebollo Arévalo en 1978 en la que se afirmaba que «la renta es la variable que condiciona en mayor medida el consumo de los españoles, de modo que la clasificación realizada considerando la renta disponible o la distribución de los gastos, proporcionan resultados similares» (1978: 73-118). En esta línea, este autor considera que la posesión de bienes de consumo, y por 
lo tanto la distribución del gasto de los españoles, se caracteriza por una jerarquización espacial que se manifiesta en el hecho que ciertas ciudades con grandes zonas industriales y un gran contingente de población ocupada en el sector servicios actúan como foco de innovación de los nuevos productos, mientras que ciudades más pequeñas adoptan el rol de «difusores» de estos productos hacia el mundo rural (Rebollo Arévalo 1978: 117), siguiendo un esquema similar al «Proceso de adopción de innovaciones» desarrollado por E.M. Rogers y E.F. Shoemaker en 1971. No obstante, en los últimos años algunos de los tradicionales «focos industriales» han desaparecido, y en su lugar emergen nuevas zonas de desarrollo como el Valle del Ebro (Navarra, La Rioja, Aragón y Tarragona) y el Denominado Arco Mediterráneo (Cataluña, Comunidad Valenciana) (Rotllant y Soy, 1993: 60-81; Alcaide, 1988).

\section{Bibliografia}

J. AlCAIDE, «El ciclo expansivo-recesivo de la economía española desde la óptica regional», en Papeles de Economía Española, n. ${ }^{\circ}$ 59-1995, pp. 2-36.

J. AlCAIDE INCHAUSTI, «El comportamiento cíclico de la economía regional entre 1986 y 1992», en Papeles de Economía Española, n. ${ }^{\circ}$ 55, FIES, CECA. Madrid 1993, pp. 2-17.

J. AlCAide InCHAusti, «Comportamiento Económico de las Comunidades Autónomas Españolas (1986-1991)», en Papeles de Economía Española, n. ${ }^{\circ}$ 51, FIES, Madrid 1992, pp. 2-33.

J. AlCAIDE, «Las cuatro Espadas económicas y la solidaridad regional», en Papeles de Economía Española, n. ${ }^{\circ}$ 34, FIES, CECA, Madrid 1988, pp. 62-81.

J. AlCAIDE y J. CuADRADO, «La economía navarra en perspectiva: una referencia a la evolución de algunas magnitudes básicas», en Papeles de Economía Española, n. ${ }^{\circ}$ 6, FIES, CECA, Madrid 1988, pp. 19-26.

F. AlCANTUd, Análisis Factorial de Correspondencias, apuntes obtenidos de un curso sobre Análisis Multivariante impartido en Bilbao en 1992 y organizado por la Asociación Vasca de Sociólogos, p. 12 o 43.

F. ANDRÉs ORIZO, Cambio Sociocultural y comportamiento económico, CIS, Madrid 1979.

F.M. ANDREWs, A Guide for Selecting Statistical Techniques for Analyzing Social Science Data, Institute for Social Research, University of Michigan, Michigan 1981.

J. ARANDa AzNAR, «La Encuesta Continua de Presupuestos Familiares», en Economistas, n. ${ }^{\circ} 17,1985$.

F. Azorín, y J.L. SÁnChez CRESPo, Métodos y Aplicaciones del Muestreo, Alianza Universidad, Madrid 1986. 
I. BALLESTER Ríos, «El equipamiento y otras características de los hogares en las Comunidades Autónomas», en Revista de Estudios de la vida local, n. ${ }^{\circ} 43$, 1984, pp. 121-140.

Banco De Bilbao y Banco Bilbao-Vizcaya, Renta Nacional de España y su distribución provincial, varios años.

J.P. BenCeCRi, L'Analyse des Données. Vol 2: Correspondances, Dunod, París 1980.

J.P. BenceCri, Practique de L'Analyse des Données. Analyse des Correspondances: exposé élémentaire, Dunod, París 1980.

J.L. Blanco FERnÁndEZ, «Hacia un indicador sintético de calidad en las Encuestas de Presupuestos Familiares», en Estadística Española, n. ${ }^{\circ} 111,1986$, pp. 5-47.

F. Calvo Gómez, Técnicas Estadísticas Multivariantes, Universidad de Deusto, Bilbao 1993.

Cámara Navarra de Comercio e Industria, Memoria económica 1992, Pamplona 1993.

S. DEl CAmpo y M. NAvarro, Nuevo análisis de la población española, Ariel, Barcelona 1987.

A. DEL CAMPO, «Una aplicación de ecología factorial al estudio de pautas especiales de segregación social en el municipio de Madrid», Ciudad y Territorio, Vol. LVII-LVIII, 3-4, 1983, pp. 137-153.

M. CAStells, La ciudad y las masas, sociología de los movimientos sociales urbanos, Alianza, Madrid 1986.

J.M. García SANTESMASES, «Análisis Factorial de Correspondencias», en J.J. SÁnchez CARrión, Introducción a las Técnicas de Análisis Multivariable aplicadas a las Ciencias Sociales, Centro de Investigaciones Sociológicas, Madrid 1984, pp. 75-105.

M.J. GreenaCre, Correspondence Analysis in practique, Academic Press, New York, 1993.

GOBIERNO DE NAVARRA, Navarra en cifras 1994, 1995.

Gobierno de Navarra, Panorama estadístico de Navarra, 1990.

Gobierno de Navarra, Población de los Ayuntamientos y Concejos de Navarra de 1900 a 1986, Pamplona 1988.

GOBIERNO VASCO, Los hábitos de compra y el comportamiento del consumidor vasco, Vitoria 1995.

H.S. HOUTHAKKER, «Ernest Engel», en Enciclopedia internacional de las ciencias sociales, Ediciones Aguilar, Madrid 1974. Vol. IV, pp. 251-252.

INE, Encuesta de Presupuestos Familiares 1990-91. Metodología, Madrid 1992.

INE, Encuesta de Presupuestos Familiares, varios años.

INE, Encuesta Continua de Presupuestos Familiares, año 1990.

INE, Encuesta de población activa: Resultados segundo trimestre de 1995.

L. Lebart, A. Morineau y J.P. FÉnelon, Tratamiento estadístico de datos, Marcombo.

J.J. LEONARDO AURTENETXE, Estructura urbana y diferenciación residencial: el caso de Bilbao, CIS-Siglo XXI, Madrid 1989.

M. Kantorowitz, Methodological Issues in Family Expenditure Surveys, Seminario Internacional de Estadística en Euskadi, Instituto Vasco de Estadística, Vitoria, octubre 1992. 
M.P. Martín GuzMán y F.J. Martín Pliego, «El consumo en servicios de las familias españolas», Papeles de Economía Española, n. ${ }^{\circ}$ 42, enero-marzo, Ed. FIES, CECA. Madrid 1990, pp. 174-192.

J.M. MúGICA, «Geografía Comercial de España: Navarra», en Distribución y Consumo, n. ${ }^{\circ}$ 5, enero-febrero 1995, pp. 82-92.

M. Navarro, La Sociedad de Consumo y su futuro, el caso de España, Ed. Ministerio de Sanidad y Consumo, Madrid 1978.

W. PetTI, «The Political Anatomy of Ireland», en C.H. Hull The economis Writings of Sir William Petty, Cambridge University Press, Cambridge 1899.

M. Rapún Gárate, P. Pascual Arzoz y P. Barritarte Zudaire, «El ciclo económico y la economía de Navarra», en Papeles de Economía Española, n. ${ }^{\circ}$ 64-1995, pp. 130.

M. RAPÚN GÁRATE, «La economía de Navarra durante el período 1985-1989», en $P a$ peles de Economía Española, n. ${ }^{\circ}$ 45, FIES, CECA, Madrid 1990, pp. 335-348.

M. RAPÚN GÁRATE, «Expansión y crisis de la economía de Navarra», en Papeles de Economía Española, n. ${ }^{\circ}$ 55, FIES, CECA, Madrid 1993, pp. 300-314.

A. Rebollo ARÉvAlo, «La transformación del consumo privado en España 19581974», en VV.AA., La Sociedad de Consumo y su futuro, el caso de España, Ed. Ministerio de Sanidad y Consumo, Madrid 1978, pp. 73-118.

A. Rebollo, La estructura del consumo de las economías domésticas en España, Instituto Nacional de Consumo, Madrid 1983.

J. RoDRíGUEZ LÓPEZ, «Algunas observaciones sobre la distribución de la renta en España», en Información Comercial Española, n. ${ }^{\text {os }}$ 528-529, agosto-septiembre 1977, pp. 222-227.

E.M. Rogers y E.F. ShoemaKer, Comunications of Innovations, Free Press, Nueva York, 1971.

E. Rotllant y A. Soy, «El papel de las ciudades en el desarollo regional. El caso español», en Papeles de Economía Española, n. ${ }^{\circ}$ 55, pp. 60-81, 1993.

A. SANZ y M. TERAN, «Las disparidades sociales regionales» en Papeles de Economía, n. ${ }^{\circ}$ 34, Madrid 1988, FIES, CECA, pp. 82-112.

M.E. Sobel, «Lifestyle Expenditures in Contemporary Amerita», en American Behavioral Scientists, vol. 26, n. ${ }^{4}$, marzo-abril 1983, pp. 521-533.

M.E. Sobel, Lifestyle and Social Structure. Academic Press, Nueva York 1981.

E. Del VAl y DE La Fuente, Lecciones de Seguridad Social, Ed. Centro de Estudios Financieros, Madrid 1986, tomo I. 